\title{
Heavy rare gases and halogens in magmas at depth: implications for elemental and isotopic fractionation
}

\author{
C. SANLOUP, C. CRÉPISSON, C. LEROY, \\ H. BuREAU, B. COCHAIN ${ }^{1}$ \\ ${ }^{1}$ IMPMC, Sorbonne Université, CNRS, Paris, France
}

High pressure silicate melts (i.e. magmas at depth), are key materials as the main transfer agents of heat and matter between Earth's reservoirs. As such, understanding their physical and chemical properties helps to constrain Earth processes.

The behaviour of heavy rare gases (xenon and krypton) and halogens (bromine and iodine) in deep melts has been investigated by means of in situ x-ray diffraction and $\mathrm{x}$-ray absorption to probe their local structure. Iodine and xenon partitioning between aqueous fluid and magma has also been investigated by in situ X-ray fluorescence.

While iodine is washed out by water degassing, Xe is retained within the melt if T exceeds $1100 \mathrm{~K}$, and then released in water [1]. This unexpected behaviour is underpinned by the P-induced reactivity of $\mathrm{Xe}$ inserts itself in the 6-membered-rings of the melt network, forming covalent bonds with the surrounding oxygens [2]. Such behaviour is only partly observed for $\mathrm{Kr}$ [3], while $\mathrm{Br}$ and I have different atomic environments [4].

$\mathrm{I}$ and Xe may thus have been fractionated by melting/cristallization at depth, whereby I would concentrate in the melt and further degas upon magma ascent, and $\mathrm{Xe}$ be retained at depth. Later cristallisation in the deep crust or mantle would preserve Xe retention within silicates [5,6], a scenario that also applies to the magma ocean stage and would provide an explanation for the atmospheric Xe missing paradox and its extreme depletion in light isotopes.

References:

[1] Leroy et al. EPSL 522:144, 2019.

[2] Leroy et al. EPSL 484:103, 2018.

[3] Crépisson et al. Chem Geol, 493:525, 2018.

[4] Cochain et al. Chem Geol 404:18, 2015.

[5] Crépisson et al. GCA 222:146, 2018.

[6] Crépisson et al. G3 20, 2019. 